\title{
Diabetogenically beneficial gut microbiota alterations in third trimester of pregnancy
}

\author{
Emmanuel Amabebe and Dilly O Anumba \\ Department of Oncology and Metabolism, University of Sheffield, Sheffield, UK \\ Correspondence should be addressed to D o Anumba: d.o.c.anumba@sheffield.ac.uk
}

\begin{abstract}
Altered gut microbiota (dysbiosis), inflammation and weight gain are pivotal to the success of normal pregnancy. These are features of metabolic syndrome that ordinarily increase the risk of type 2 diabetes in non-pregnant individuals. Though gut microbiota influences host energy metabolism and homeostasis, the outcome (healthy or unhealthy) varies depending on pregnancy status. In a healthy pregnancy, the gut microbiota is altered to promote metabolic and immunological changes beneficial to the mother and foetus but could connote a disease state in non-pregnant individuals. During the later stages of gestation, metabolic syndrome-like features, that is, obesity-related gut dysbiotic microbiota, increased insulin resistance, and elevated pro-inflammatory cytokines, promote energy storage in adipose tissue for rapid foetal growth and development, and in preparation for energy-consuming processes such as parturition and lactation. The origin of this gestation-associated host-microbial interaction is still elusive. Therefore, this review critically examined the host-microbial interactions in the gastrointestinal tract of pregnant women at late gestation (third trimester) that shift host metabolism in favour of a diabetogenic or metabolic syndrome-like phenotype. Whether the diabetogenic effects of such interactions are indeed beneficial to both mother and foetus was also discussed with plausible mechanistic pathways and associations highlighted.
\end{abstract}

\section{Lay summary}

In non-pregnant women, increased blood glucose, fat accumulation, and prolonged immune response lead to obesity and diabetes. However, during the later stages of pregnancy, the changes in the body's metabolism described previously do not lead to disease, instead pregnancy facilitates the storage of sufficient energy in fat cells for rapid growth and development of the foetus. The excess energy stores also prepares the mother for labour and breastfeeding. This review examines the role of the normal bacteria in the digestive tract in this beneficial energy accumulation and transfer between the mother and foetus without leading to obesity, diabetes and hypertension in pregnancy.

Key Words: - pregnancy $\quad$ gut $\quad$ microbiota $\quad$ diabetes $\quad$ metabolic syndrome $\quad$ inflammation

Reproduction and Fertility (2021) 1 R1-R12

\section{Introduction}

Metabolic syndrome (MetS) is characterised by a spectrum of clinical findings (phenotypes) including hyperglycaemia, insulin resistance, increased adiposity, and chronic subclinical inflammation, that aggravate an individual's risk of type 2 diabetes mellitus (T2DM) (Koren et al. 2012). Though there are several definitions of MetS, a unified international definition incorporating the definition by the National Cholesterol Education Program Adult 
Treatment Panel III (NCEP/ATPIII) has been recommended. The definition also proposed the use of population-specific waist circumference thresholds to determine obesity (Samson \& Garber 2014). The harmonised definition of MetS issued in 2009 is presented in Table 1.

The over 100 trillion resident bacteria in the human gastrointestinal tract (gut microbiota) are crucial to human metabolism, immunity and overall health (Edwards et al. 2017, Amabebe et al. 2020). Due to its immense contribution to the maintenance of homeostasis, the gut microbiota has been described as a microbial organ (Edwards et al. 2017, Amabebe et al. 2020). The constituent microorganisms, which are acquired at birth, evolve through a mutualistic relationship with the host (Liu et al. 2017). This microbial evolution continues throughout life influenced by several factors including genetic factors, mode of delivery, maternal and infant dietary habit, age, environment and pregnancy (Saad et al. 2016, Edwards et al. 2017, Ponzo et al. 2019).

Pregnancy induces immunological and metabolic changes manifested as immune tolerance that allow implantation and placentation; and insulin resistance to support growth of the foetus (Crusell et al. 2018). Because the later stage (especially third trimester) of pregnancy is characterised by MetS-like features, that is, maternal hyperglycaemia, increased adiposity, high levels of circulating proinflammatory chemocytokines and insulin resistance; it has been described as a diabetogenic state (Mor \& Cardenas 2010). These physiological changes can even lead to gestational diabetes in high-risk women (Crusell et al. 2018).

Table 1 Harmonised definition of metabolic syndrome.

\begin{tabular}{|c|c|}
\hline & Clinical feature \\
\hline $\begin{array}{l}\text { Criteria/no. of risk } \\
\text { factors }\end{array}$ & $\geq 3$ of the risk factors below \\
\hline Obesity & WC: population-specific values* \\
\hline Hyperglycaemia & $\mathrm{FPG} \geq 5.6 \mathrm{mmol} / \mathrm{L}(100 \mathrm{mg} / \mathrm{dL})$ or treated \\
\hline Hypertension & $\begin{array}{l}\mathrm{SBP} \geq 130 \mathrm{mmHg} \\
\mathrm{DBP}>85 \mathrm{mmHg} \text { or treated }\end{array}$ \\
\hline Dyslipidaemia & $\begin{array}{l}\mathrm{HDL}-\mathrm{C}:<1.0 \mathrm{mmol} / \mathrm{L}(40 \mathrm{mg} / \mathrm{dL})(\mathrm{M}) \\
<1.3 \mathrm{mmol} / \mathrm{L}(50 \mathrm{mg} / \mathrm{dL})(\mathrm{F}) \\
\mathrm{TG}: \geq 1.7 \mathrm{mmol} / \mathrm{L}(150 \mathrm{mg} / \mathrm{dL}) \text { or treated }\end{array}$ \\
\hline
\end{tabular}

This definition is a joint statement issued in 2009 by the International Diabetes Federation, American Heart Association/National Heart, Lung and Blood Institute, the World Heart Federation, the International Atherosclerosis Society, and the International Association for the Study of Obesity.

*Population-specific waist circumference values can be found in Samson and Garber (2014).

DBP, diastolic blood pressure; F, female; FPG, fasting plasma glucose; HDLC, high-density lipoprotein-cholesterol; M, male; SBP, systolic blood pressure; TG, triglyceride; WC, waist circumference.
Considering the interrelationship between pregnancy, obesity and diabetes through their common features, that is, low-grade systemic inflammation, altered gut microbial composition, insulin resistance and hyperglycaemia, the evaluation of these features and their association especially in relation to maternal and foetal health is of great importance. Interestingly, the MetS-like phenotype observed in later gestation is not overtly harmful to the mother and foetus in most cases. In fact, it has been described as beneficial and necessary for a successful parturition and postpartum health (Collado et al. 2008, Santacruz et al. 2010, Koren et al. 2012, Edwards et al. 2017, Liu et al. 2017). However, the mechanistic link between these diabetogenic phenotypes and periparturition health and safety of the fetomaternal unit without leading to gestational diabetes or other complications, is still unresolved and deserves more attention. Therefore, this review critically examined the host-microbial interactions in the gastrointestinal tract of pregnant women at late gestation (third trimester) that shift host metabolism in favour of a diabetogenic or metabolic syndrome-like phenotype. Whether the diabetogenic effects of these interactions are indeed beneficial to both mother and foetus was also discussed with plausible mechanistic pathways and associations highlighted.

\section{Pregnancy-induced microbial changes}

The endocrine and physical changes that accompany pregnancy trigger an array of anatomical, physiological and biochemical alterations that affect every organ of the body (Prince et al. 2014). The microbiome of different body sites including the vagina and gut which are in a continuous crosstalk (Amabebe \& Anumba 2020) also exhibit immunological and metabolic changes associated with the progression of normal, healthy gestation. At the onset of pregnancy, the immune system represses itself to allow and support implantation of the foetus, and restrengthens towards mid and later stages of pregnancy to prevent invasion by pathogens (Edwards et al. 2017). This 'rewiring of immune system' throughout pregnancy induces a low-grade inflammation at the mucosal surfaces of gut, oral cavity, vagina and placenta. This leads to structural and compositional changes in the microbiota of these body sites (Edwards et al. 2017).

The vaginal microbiota at late gestation is similar to that of a non-pregnant (non-menstruating and bacterial vaginosis, BV-negative) state characterised by a decrease in $\alpha$-diversity (species richness - within individual diversity or amount of different species detected in the vaginal sample),

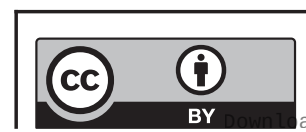

This work is licensed under a Creative Commons Attribution 4.0 International License. 
and a corresponding increase in Lactobacillus spp. (Kaur et al. 2020). This resemblance is believed to trigger the changes that are associated with parturition. As a prerequisite for a successful pregnancy, the vaginal microbiome shifts in favour of Lactobacillus spp. dominance, becomes more stable and less diverse. Of course, this is not without the support of rising oestrogen, increased vaginal epithelial glycogen accumulation, and increased acidity (Amabebe \& Anumba 2018, 2020, Kaur et al. 2020).

Both Lactobacillus crispatus and Lactobacillus jensenii increase as part of the vaginal microbiomial stability associated with gestation. These species metabolise glycogen hydrolysates to produce lactic acid to propagate their growth and replication. This is facilitated by high levels of oestrogen that stimulates glycogen accumulation in vaginal epithelial cells and epithelial-derived $\alpha$-amylase that depolymerises the $\alpha$-glucan molecules (Amabebe \& Anumba 2018, Tester \& Al-Ghazzewi 2018). In addition to the lactic acid, the lactobacilli produce hydrogen peroxide and bacteriocins that inhibit the growth of BV-associated pathogens (Amabebe \& Anumba 2018, Tester \& Al-Ghazzewi 2018), thereby preventing infection associated spontaneous preterm birth (Prince et al. 2014).

The gestation-associated changes in the vaginal microbiota also have postnatal benefits to the offspring. Lactobacillus johnsonii is primarily found in the upper gut where it sustains its survival and persistence by catalysing the hydrolysis (i.e. release of taurine and glycine) and uptake of bile through its bile salt hydrolase and transporters (Pridmore et al. 2004). It inhibits the growth and survival of other lactobacilli and enterococci in the gut through the production of bacteriocin - Lactacin F (Abee et al. 1994). These properties aid postnatal digestion of breast milk by neonates inoculated with vaginal $L$. johnsonii at birth (Prince et al. 2014).

\section{Non-pregnant gut microbiota}

Firmicutes and Bacteroides, which constitute $>95 \%$ of the bacterial population, dominate the physiologic gut microbiota in the non-pregnant state. Other bacteria phyla/divisions present in small proportions include Proteobacteria, Fusobacteria, Actinobacteria and Verrucomicrobia (Amabebe \& Anumba 2020, Amabebe et al. 2020). These microorganisms ferment non-digestible dietary fibres to produce metabolites (short chain fatty acids, SCFAs) that maintain intestinal barrier integrity and prevent leakage of bacteria and bacterial products such as lipopolysaccharide (LPS) in to systemic circulation
(Amabebe \& Anumba 2020, Amabebe et al. 2020). The microbiota is distributed throughout the gut varying according to region and determined by $\mathrm{pH}$, nutrient, and oxygen availability (Saad et al. 2016). Overall, the gut microbes influence energy utilisation, hormonal, immunologic and metabolic health of the host. Alterations in the gut microbial composition is linked to metabolic and immunologic conditions including obesity, T2DM, inflammatory bowel disease (IBD), irritable bowel syndrome, allergies, pre-eclampsia, gestational diabetes, etc. Detailed review of the role of gut microbiota in health and disease can be found in the following reports (Canfora et al. 2015, Saad et al. 2016, Chambers et al. 2018, Tungland 2018, Lazar et al. 2019, Parada Venegas et al. 2019, Amabebe \& Anumba 2020, Amabebe et al. 2020).

\section{Gut microbiota changes in late gestation}

At the beginning of pregnancy, the gut microbiota profile resembles that of a healthy non-pregnant woman characterised by predominance of Firmicutes (particularly Clostridiales, Faecalibacterium prausnitzii) over Bacteroidetes (Walters et al. 2014). Subsequently, gut microbiota is greatly altered over the course of pregnancy. Maternal gut function and microbiota are altered by the unique physiobiochemical, inflammatory and immune responses that occur as pregnancy advances. These alterations permit changes in maternal metabolism and weight required to maintain pregnancy and survival of the foetus after delivery (Koren et al. 2012, Edwards et al. 2017).

In a European cohort, the gut microbiota species richness or within individual $\alpha$-diversity (Ponzo et al. 2019) decreased from the first to third trimester with overall increase in Proteobacteria (Enterobacteriaceae, Escherichia coli) and Actinobacteria (Propionibacterium) (Fig. 1); whereas there was an increase in inter-subject/sample/site $\beta$-diversity. A reduction in the health-promoting, butyrogenic and antiinflammatory Faecalibacterium prausnitzii is also observed by the third trimester (Koren et al. 2012, Garcia-Mantrana \& Collado 2016, Nuriel-Ohayon et al. 2016). This is similar to the microbial composition observed in non-pregnant adults with MetS (Koren et al. 2012). F. prausnitzii abundance is inversely related with low-grade inflammation, plasma glucose and diabetes; with a capacity to predict the risk of T2DM (Ferrocino et al. 2018). These microbial alterations permit a chronic low-grade inflammation at the mucosal surfaces of the gut; and may be influenced by diet, antibiotic therapy, pre-pregnancy BMI and gestational diabetes. However, such microbial alterations are usually

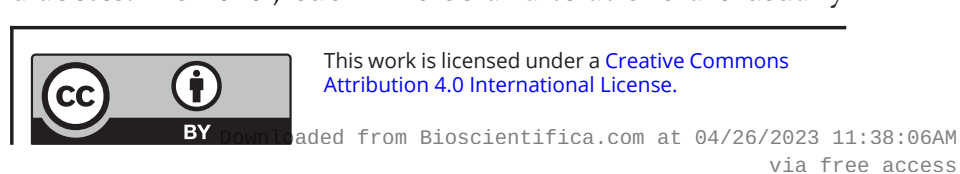



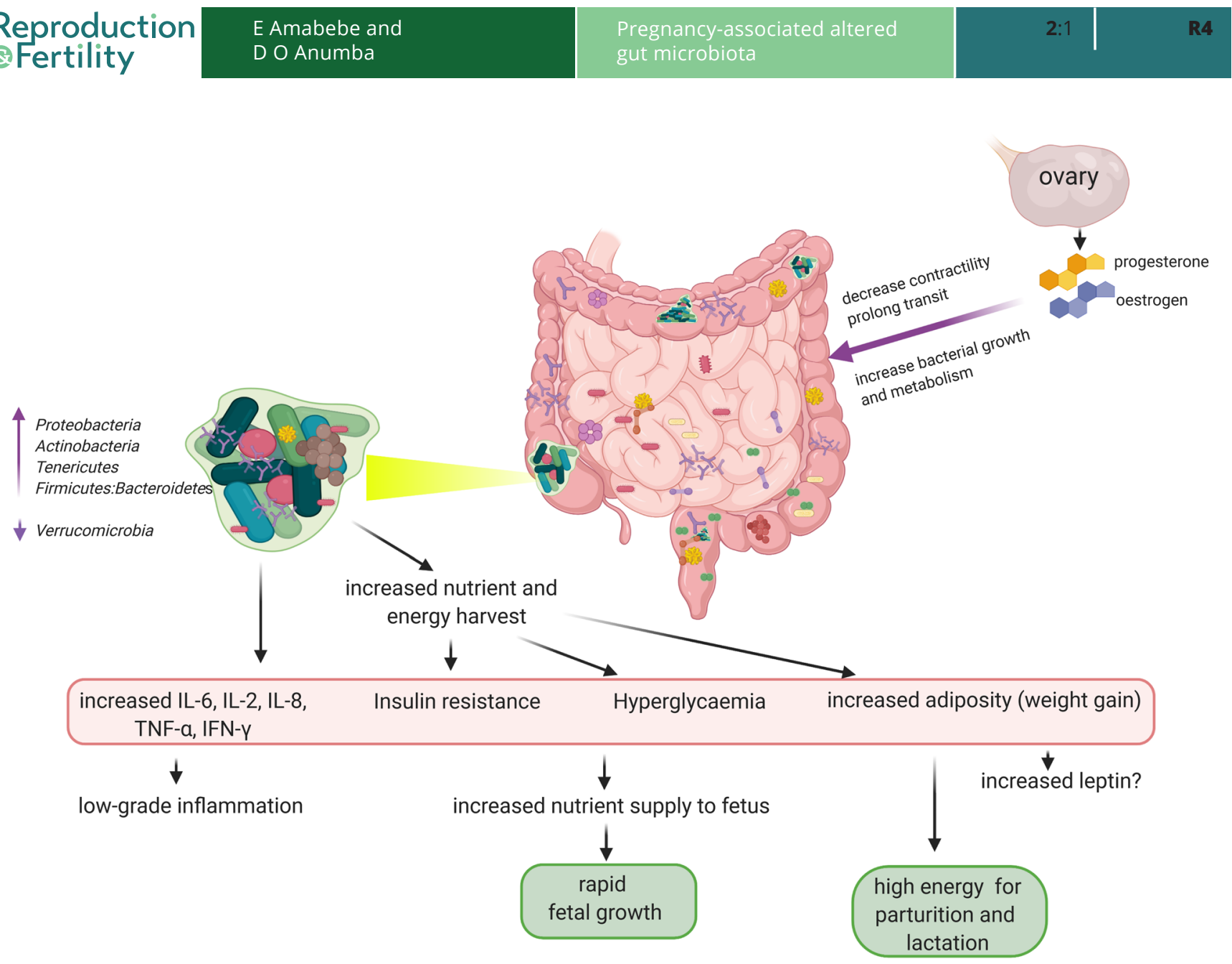

Figure 1 Third trimester-associated diabetogenically beneficial gut microbiota. As pregnancy advances, hormonal and immunological changes increase nutrient and energy harvest from the gut. These changes are induced by increased oestrogen and progesterone that inhibit gastrointestinal contractility and prolong transit providing a suitable environment (substrates) for energy-harvesting microbes. The consequent dysbiosis triggers a low-grade inflammatory state propagated by pro-inflammatory chemocytokines leading to insulin resistance and hyperglycaemia. This diabetogenic phenotype preferentially supplies abundant nutrients to the maturing foetus and prepares the mother for energy-demanding processes of parturition and lactation through the accumulation of fat and weight gain. There are also possibilities of adiposity-induced increased leptin and perhaps leptin resistance as seen in obesity. Though these are typical features of metabolic syndrome, they are not usually detrimental to maternal and fetal health in the later stage of gestation. Created with Biorender.com.

not related to these factors (Koren et al. 2012, Gohir et al. 2015, Mesa et al. 2020).

Furthermore, the microbial changes and adaptations may be gene and/or environment-dependent as other phyla were either decreased (i.e. Verrucomicrobia) or increased (i.e. Tenericutes) (Fig. 1) in the third trimester in a pregnant Southern Chinese cohort. Moreover, Bacteroidetes (acetate and propionate producers) were the most abundant bacteria in these women, while Firmicutes (major butyrate producers) reduced relatively (Liu et al. 2017). Butyrate has shown more anti-inflammatory activities than acetate and propionate, hence, frequently used in the treatment of inflammatory bowel disease (IBD, ulcerative colitis and Crohn's disease) and colorectal cancers (Cox et al. 2009, Mirmonsef et al. 2011, 2012, Koh et al. 2016, Rivière et al. 2016, Parada Venegas et al. 2019).
Alas, the intestinal levels of SCFAs were not determined in this Chinese cohort.

Animal models have demonstrated Verrucomicrobia is associated with poor digestion and absorption of nutrients (mice) (Preidis et al. 2015), while increased Tenericutes enhance nutrient digestion and absorption (i.e. increased energy harvest) in pigs (Niu et al. 2015). The increase in Tenericutes and decrease in Verrucomicrobia, and the corresponding enhanced energy harvest observed in late gestation of some Chinese women may be a similar adaptation to the high energy demands required for foetal growth and maturation, parturition and lactation (Fig. 1) (Liu et al. 2017). The strengthened digestive and absorptive capacity could be an adaptive mechanism required for the higher energy and metabolic processes in late pregnancy (Liu et al. 2017).

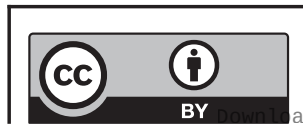

This work is licensed under a Creative Commons Attribution 4.0 International License. 


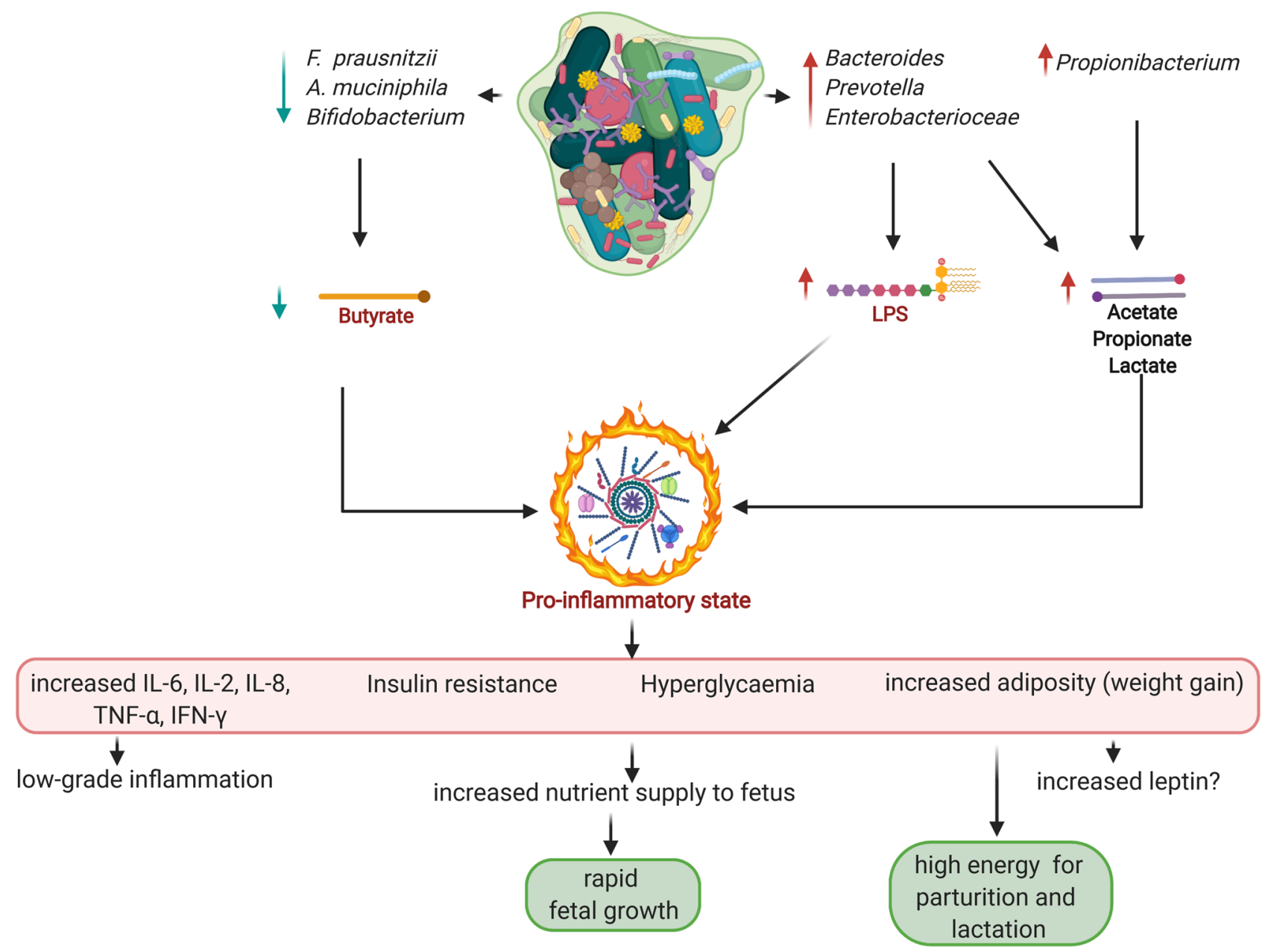

Figure 2 Gut microbial-metabolite profile in third trimester of normal pregnancy. The low-grade pro-inflammatory state that leads to glucose dysmetabolism and insulin resistance albeit non-injurious to mother and foetus, may be a constellation of high lipopolysaccharide (LPS) from the predominating Gram-negative species - Bacteroides, Prevotella and Escherichia coli; and low butyrate levels due to a decline in butyrogenic species - Faecalibacterium, Akkermansia, Bifidobacterium. High acetate, propionate and lactate produced by the Gram-negative species in collaboration with Propionibaterium are unable to inhibit the pro-inflammatory state. Hence, the declining butyrate level may be the determinant factor. Created with Biorender.com.

Additionally, the low abundance of probiotic Firmicutes such as Coprococcus catus (Liu et al. 2017) and F. prausnitzii (which lack LPS) (Koren et al. 2012, Garcia-Mantrana \& Collado 2016, Nuriel-Ohayon et al. 2016), in relation to Bacteroidetes (that possess high LPS) may lead to elevated LPS production and subsequent inflammation that may promote the MetS-like phenotype at late gestation (Fig. 2) (Liu et al. 2017). However, this study (Liu et al. 2017) did not indicate the species of the Bacteroidetes phyla that may be associated with the proposed elevated LPS.

In the non-pregnant state, gut bacterial LPS can induce a chronic subclinical inflammatory response and obesity, leading to insulin resistance by activation of tolllike receptor (TLR)-4 (Saad et al. 2016). It is noteworthy that some genera and species of the Bacteroidetes phyla are obesogenic (e.g. Prevotella spp.) (Amabebe et al. 2020) and diabetogenic (e.g. Bacteroides fragilis) (Mancuso et al. 2005), while others are anti-obesogenic (e.g. Bacteroides thetaiotaomicron) (Kobyliak et al. 2016, Amabebe et al. 2020). This LPS induced Bacteroidetes/Firmicutes relationship with diabetogenic phenotype at later gestation requires resolution down to species level for a more comprehensive understanding of the mechanistic pathways.

\section{Firmicutes:Bacteroidetes ratio}

Non-pregnant and first trimester pregnant women share similar gut microbial diversity (Santacruz et al. 2010). However, as pregnancy advances, the abundance of 
inflammation-associated gut bacterial species increases in most women (Santacruz et al. 2010). Maternal pregestational BMI and weight gain over gestation can also influence the shift in gut microbiota (Collado et al. 2008, Santacruz et al. 2010, Zacarías et al. 2018). Lowgrade systemic inflammation, insulin resistance and a dysbiotic gut microbiota are characteristic of increased adiposity as seen in obesity and pregnancy especially the third trimester (Zacarías et al. 2018). Similar to the obesity and MetS-related symptoms in non-pregnant state, the ratio of potentially pathogenic (pro-inflammatory) Firmicutes to Bacteroidetes increases in relation to gestational age especially in obese women. Therefore, at third trimester, there could be more Firmicutes than Bacteroidetes depending on the mother's BMI. Elevated levels of potentially pathogenic (pro-inflammatory) Firmicutes (e.g. Clostridium perfringens) and ultimately higher Firmicutes:Bacteroidetes ratio was recently observed in overweight and obese pregnant women in the third trimester compared to their normal weight counterparts (Zacarías et al. 2018). However, the anti-inflammatory health-promoting Firmicutes, that is, F. prausnitzii and other butyrate producers of the Lachnospiraceae family are decreased in obesity (Chakraborti 2015) and IBD (Rivière et al. 2016). The Firmicutes are also high energy harvesters, that is, they metabolise non-digestible polysaccharides for example, starch, glycogen, etc., to release energy (Santacruz et al. 2010). It was suggested that this MetSlike host-microbial interaction might be importantly beneficial in pregnancy, for energy storage, foetal growth, parturition and lactation. The plausible mechanisms underlying these speculations are; elevated dietary energy extraction efficiency; and disrupted host-microbial interactions that induce metabolic inflammation. Taken together, gestation-associated changes (imbalance) in gut microbiota with corresponding increase in energy harvesting microbes could induce metabolic inflammation (Koren et al. 2012).

However, as shown in Fig. 1, it appears the relative abundance of $F$. prausnitzii, an anti-inflammatory Firmicutes that constitutes a substantial part of the normal gut microbiota along with other butyrateproducing Firmicutes (Lopetuso et al. 2013, Chakraborti 2015, Amabebe \& Anumba 2020) influences the overall immunometabolic phenotype observed in later gestation regardless of the Firmicutes:Bacteroidetes ratio. That is, even though the Firmicutes:Bacteroidetes ratio remains high in the later stages of gestation as seen in obesity, a low $F$. prausnitzii abundance could promote a pro-inflammatory state similar to MetS (Koren et al. 2012, Garcia-Mantrana
\& Collado 2016, Nuriel-Ohayon et al. 2016, Ferrocino et al. 2018).

\section{Gut bacterial load and weight gain in pregnancy}

Pregnant overweight women that harbour a high amount of energy harvesting microbes usually supply high amount of energy to the foetus. This could lead to a high birth weight and increased risk of complications to both the mother and newborn (Collado et al. 2008). The gut bacterial load increases throughout pregnancy irrespective of maternal body weight. Specifically, Bacteroides. fragilis and Staphylococcus have been shown to increase with weight gain as pregnancy advances in normal weight women (Collado et al. 2008, Santacruz et al. 2010). Furthermore, maternal pregestational BMI is inversely related to gestational weight gain. For example, obese women show decreased gestational weight gain (Broskey et al. 2017, Zacarías et al. 2018); and a possibly related decrease in Bacteroides by third trimester (Zacarías et al. 2018).

At 30-35 weeks of gestation, normal weight women showed higher Bacteroides and lower Faecalibacterium compared to overweight women (Zacarías et al. 2018). They also had lower Coprococcus, Blautia, Catenibacterium and Actinomyces compared to obese pregnant women at the same gestation (Zacarías et al. 2018). Normal pregestational BMI is associated with reduced Firmicutes - Blautia, Coprococcus, Anaerostipes, Holdemenia, and Bulleidia; and increased Bacteroides, Coriobacteriaceae (Collinsella, Eggerthella, Atopobium) and Methanobrevibacter at third trimester. High pregestational BMI (i.e. obesity) is associated with a high amount of Lachnospiraceae. These unique microbial compositions could partly explain the third trimester-associated elevated Firmicutes:Bacteroidetes ratio observed in overweight/obese women compared to their normal weight counterparts (Zacarías et al. 2018).

B. fragilis also has an LPS-dependent pathologic association with diabetes (Mancuso et al. 2005). Methanobrevibacter is known to enhance energy extraction by utilisation of $\mathrm{H}_{2}$ produced by bacterial fermentation of dietary fibres (Amabebe et al. 2020). Coriobacteriaceae adversely affect plasma cholesterol metabolism, that is, positively correlated with non-HDL-C (Clavel et al. 2014); and are enriched in obese animals (Clarke et al. 2012). Members of this family have been identified in cases of IBD, periodontitis, colon cancer, diabetes and bacteraemia. Enterobacteriaceae (E. coli) also increase with weight gain as gestation advances (Santacruz et al. 2010). These gestational weight-associated bacterial community

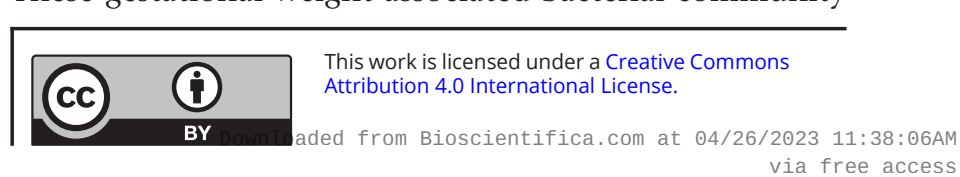


alterations are usually associated with lower within subject $\alpha$-diversity (Koren et al. 2012, Stanislawski et al. 2017, Zacarías et al. 2018), pro-inflammation, insulin resistance and hyperglycaemia (Qin et al. 2018).

In addition, Bifidobacterium and Akkermansia muciniphila decrease with weight gain over gestation (Collado et al. 2008, Santacruz et al. 2010). High bifidobacteria and $A$. muciniphila correlate with reduced inflammation, improved glucose tolerance and insulin sensitivity (Vyas et al. 2019, Amabebe et al. 2020, Xu et al. 2020). Therefore, low amounts of these butyrate-producing species as observed with increased maternal weight gain towards the third trimester may contribute to the inflammatory processes and MetS-like phenotype associated with this stage of pregnancy (Fig. 2) (Collado et al. 2008).

Changes in gut microbiota influence host gestational weight gain through increased glucose and fat absorption, enhanced secretion of fasting-induced adipocyte factor (FIAF), induction of catabolic pathways, and stimulation of a low-grade pro-inflammatory state (Bäckhed et al. 2007, Collado et al. 2008, Koren et al. 2012). Taking the studies of Collado et al. (2008), Santacruz et al. (2010), Koren et al. (2012) and Liu et al. (2017) together, the increased energy extraction and pro-inflammation observed in the third trimester of normal pregnancy may be the result of a synergy between Proteobacteria (Enterobacteriacaea), Actinobacteria (Propionibacterium), Tenericutes and some Firmicutes (non-butyrate producers). The inflammation is further promoted by the reduction in Faecalibacterium, Bifidobacterium and A. muciniphila and increase in obesogenic/diabetogenic Bacteroidetes such as Prevotella spp. and B. fragilis with resultant high circulating LPS levels and insulin resistance (Figs 1 and 2). This diabetogenically favourable gut microbial synergy at the later stage of gestation warrants further investigation especially at bacterial genus, species as well as metabolite levels. Besides, the type and level of metabolites produced by the bacteria may be the ultimate determinant of the observed inflammatory status.

Nevertheless, gut microbiota may also contribute to gestational dysmetabolism and inflammation through other yet unresolved mechanisms. Diseaseassociated correlations between specific taxa and gestational metabolic markers have been identified. For example, high amount of lactate-producing Collinsella (Actinobacteria) is associated with increased circulating levels of insulin, homeostatic model assessment of insulin resistance (HOMA-IR), triglycerides, and very-low-density lipoproteins. Similarly, Sutterella (Proteobacteria) and C-reactive protein (CRP) - a marker of chronic low-grade systemic inflammation as seen in obesity, diabetes, heart disease and colorectal cancer (Zacarías et al. 2018); Ruminococcaceae/Lachnospiraceae and leptin; Bacteroidaceae and ghrelin; Coprococcus and gastrointestinal polypeptide (GIP) are directly related. In addition, high abundance of Blautia spp. is associated with low insulin, HOMA-IR and HbA1c; increase in Faecalibacterium or Faecalibacterium/Fusobacterium ratios is associated with low fasting blood glucose; and high butyrate-producing Odoribacter (Bacteroidetes) is associated with low arterial blood pressure in overweight pregnant women. Similar inverse relationships have been reported for Ruminococcaceae and GIP, as well as Prevotellaceae and ghrelin (Ferrocino et al. 2018, Ponzo et al. 2019).

\section{Elevated production of pro-inflammatory mediators}

In normal pregnancy, there is increase in body fat in early gestation and subsequent reduced insulin sensitivity (Barbour et al. 2007). Reduced insulin sensitivity correlates with elevated levels of circulating chemocytokines for example, TNF- $\alpha$, IL- 6 and IL-8 (Kirwan et al. 2002) that trigger obesity-associated metabolic inflammation (Gregor \& Hotamisligil 2011). Though these inflammatory responses are detrimental to long-term health in the obese state, increased adiposity, hyperglycaemia and decreased insulin sensitivity favour foetal growth and prepare the mother's body for the energetic demands of parturition and lactation. Therefore, the pro-inflammatory state is beneficial in the context of a normal pregnancy (Di Cianni et al. 2003, Lain \& Catalano 2007, Nelson et al. 2010, Koren et al. 2012).

The increased abundance of gut Proteobacteria as pregnancy advances is associated with significantly higher levels of IFN- $\gamma$, IL-2, IL-6, and TNF- $\alpha$ in third trimester than in the first (Fig. 1) (Koren et al. 2012). The serum, adipose and placental tissue levels of these pro-inflammatory mediators are seen to increase later in pregnancy of humans with the mucosal surfaces of the entire gastrointestinal tract reflecting a low-grade inflammatory state (Cani et al. 2012). Transfer of gut microbiota from healthy women in third-trimester of pregnancy to a gemfree mice stimulated increased adiposity, insulin resistance and increased production of pro-inflammatory cytokines including IL-1 $\beta$, IL-2, IL-5, IL-6 and GM-CSF compared to mice inoculated with microbiota from healthy women in the first trimester of pregnancy (Koren et al. 2012). This is somewhat akin to the impact observed when gut

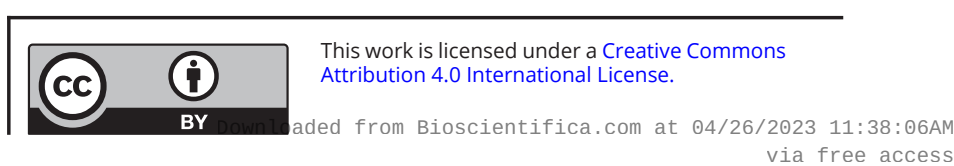


microbiota from non-pregnant obese human donors are transferred to gem-free mice (Ridaura et al. 2013). That this gestation-related pro-inflammatory and diabetogenic gut microbiota is beneficial to foetal development and prepares the mother for postnatal nurturing is thought provoking. Understanding the factors (genetic or otherwise) underlying these fetomaternal microbial, immune and metabolic interactions could guide diagnostic and therapeutic strategies for conditions such as gestational diabetes and pre-eclampsia.

Zacarías et al. (2018) have recently demonstrated another evidence in support of the association of pregnancy-related gut microbial changes and inflammation. Similar to obesity (Turnbaugh et al. 2009, Fu et al. 2015), third trimester gut microbiota showed lower $\alpha$-diversity (similar to Koren et al. 2012) that was associated with increased circulating high-sensitive CRP (hs_CRP) and haptoglobin - both confirmed markers of inflammation characteristic of metabolic diseases such as obesity, diabetes, cancers, etc. Reduced gut microbial diversity and richness is associated with increased adiposity and inflammatory profile (Cotillard et al. 2013, Verdam et al. 2013). In general, serum levels of both inflammatory mediators increased with high amounts of Firmicutes - Blautia, Coprococcus, Anaerostipes, Holdemenia, and Bulleidia; and reduced amounts of Bacteroides, Coriobacteriaceae and Methanobrevibacter. Individually, hs CRP was inversely related to Faecalibacterium. Haptoglobin which also acts as a chemoattractant for macrophages in adipose tissue increased with low Firmicutes (normal weight women) and low Bacteroidetes (overweight women). Haptoglobin was also directly related to Firmicutes in overweight women; and generally to Ruminococcus gnavus (a member of Clostridium cluster XIVa - mucosal butyrate producers) (Zacarías et al. 2018). The mechanisms underpinning these observations and subsequent clinical implications are poorly understood. Whether they are exclusively related to the beneficial diabetogenic state of third trimester needs to be determined by further in vivo and in vitro experiments.

\section{Effect of oestrogen and progesterone on gut function}

Some researchers have hypothesised that high levels of pregnancy-related hormones that increase gut transit time could be an adaptation that facilitates enhanced nutrient and energy harvest, thereby promoting weight gain in pregnancy (Edwards et al. 2017). This is based on the effect of the significant rise in ovarian hormones during prenatal and postpartum periods on intestinal contractility and transit (Mulak et al. 2014). Physiologic hyperoestrogenaemia and hyperprogesteronaemia as seen in later stages of pregnancy can reduce gastrointestinal contractility and prolong transit (Fig. 1) (Mulak et al. 2014). This can encourage enhanced nutrient and energy extraction especially in the abundance of energy harvesting microbes such as Tenericutes and some Firmicutes. These symptoms are similarly observed in the context of irritable bowel syndrome (IBS) (Mulak et al. 2014).

Furthermore, oestrogen and progesterone can directly modulate gut microbiota composition, bacterial metabolism, growth and expression of virulence factors (Mulak et al. 2014). It appears the ovarian hormones participate in creating a suitable environment for gut microbial-nutrient interaction that leads to increased nutrient digestion and absorption (Fig. 1). This manifests as weight gain for both mother and foetus and a MetS-like phenotype.

Ovarian hormones also influence insulin sensitivity during pregnancy (Bruns \& Kemnitz 2004). Maternal insulin resistance is associated with increasing levels of progesterone and oestrogen in the third trimester. This supports rapid foetal growth by promoting the preferential transfer of carbohydrates to the foetus (Buchanan 1991). The foetus also produces high amounts of insulin in response to excess glucose, and foetal insulin stimulates growth in utero (Bruns \& Kemnitz 2004). Some mothers are unable to compensate adequately for this hormone-induced insulin insensitivity, and gestational diabetes ensues (Buchanan 1991). On the other hand, in a vast majority of pregnant women, a compensatory mechanism(s) ensures a reversal to normal insulin sensitivity and glucose tolerance indicating a beneficial effect of the hormone-related diabetogenic phenotype. Women who develop gestational diabetes are unable to produce an adequate amount of insulin to maintain euglycaemia, and exhibit greater insulin resistance with resultant glucose intolerance (Hod et al. 2015).

\section{Microbiota-metabolite profile}

Another plausible mechanistic pathway to the beneficial diabetogenic phenotype characteristic of the later stage of gestation is the microbial-induced disequilibrium in production of SCFAs - acetate, butyrate and propionate (Fig. 2). SCFAs are derived from intestinal bacterial 
fermentation of indigestible dietary fibres. They are energy substrates and regulate satiety and food intake. They regulate glucose and energy homeostasis and metabolism through both local (intestinal) and systemic (brain, liver, adipocytes, skeletal muscle etc.) effects. They also enhance intestinal epithelial barrier integrity, and inhibit bacterial translocation and inflammation. The roles of SCFAs in control of body weight, obesity and diabetes in non-pregnant state is well defined (Canfora et al. 2015, Saad et al. 2016, Vyas et al. 2019, Amabebe \& Anumba 2020, Amabebe et al. 2020).

Butyrate produces more potent anti-inflammatory responses compared to acetate and propionate (Cox et al. 2009, Mirmonsef et al. 2011, 2012, Koh et al. 2016, Rivière et al. 2016, Parada Venegas et al. 2019). The dysbiotic microbiota at late gestation displays a local dysmetabolism characterised by reduced butyrate levels relative to acetate, propionate and lactate. In conjunction with increased circulating LPS from the Gram-negative bacterial species (e.g. Bacteroides, Prevotella and Enterobacteriaceae), decreased butyrate could create an immune imbalance in favour of a pro-inflammatory state with an elaborate systemic dysmetabolism - insulin resistance, hyperglycaemia and increased weight gain, albeit not injurious in this context (Fig. 2).

The declining butyrate level might be the determining factor that perpetuates the pro-inflammatory state, as this state is maintained despite an increase in acetate, propionate and lactate that are somewhat antiinflammatory. For example, Proteobacteria with adherentinvasive capacities (E. coli, Campylobacter concisus, and enterohepatic Helicobacter) are active components of IBD (Mukhopadhya et al. 2012); and E. coli colonisation alone induce sufficient macrophage infiltration of white adipose tissue, reduced insulin sensitivity and glucose intolerance in germ-free mice (Caesar et al. 2012). Butyrate has found extensive therapeutic use against IBD due to its potent anti-inflammatory actions compared to the other SCFAs (Silva et al. 2018, Chen \& Vitetta 2020). Chen and Vitetta (2020) have recently highlighted the clinical utility of the extensive anti-inflammatory properties of butyrate and advocated butyrate supplementation (singly or in combination with other anti-inflammatory agents) as a practical approach in treating IBD. These evidence highlight the importance of butyrate in the inflammation equation linked to gestation-induced gut dysbiosis. These hypotheses are yet to be explored in relation to the physiology of pregnancy and parturition. However, longitudinal metabolomics of the gut microbiome in pregnant women and postnatally could provide more insight on the benign effect of this dysbiotic, dysmetabolic and inflammatory phenotype, instead of an outright disease state.

\section{Conclusion and future perspectives}

Despite its devastating clinical appearance, the prognosis of MetS-related features may not necessarily be deleterious to the host or foetus in the case of pregnancy. During normal pregnancy, the disease-associated gut microbial dysbiosis and related immunological responses that increase the risk of obesity and diabetes create an interestingly favourable metabolic environment that preferentially supplies sufficient nutrients for rapid foetal growth and development while preparing the mother for parturition and lactation. At third trimester, the gut microbiotainduced energy metabolism that encourages energy efficacy and storage (Garcia-Mantrana \& Collado 2016) shifts in favour of the foetus who requires an adequate supply of nutrients for rapid growth. Concomitantly, a transient maternal reduced insulin sensitivity creates a benign diversion of nutrients for foetal use instead. Similar to obesity, the insulin resistance is triggered by a low-grade systemic inflammatory state. The increased weight gain (increased fat and energy storage) in the mother supplies the energy required for delivery and lactation.

In some cases, these benign processes can become pathologic leading to gestational diabetes and preeclampsia. However, a potential overt inflammation and dysmetabolism is usually averted by yet unresolved compensatory mechanisms. The gut microbiota associated with this beneficial diabetogenic phenotype at late gestation is believed to be characterised by an increase in energy harvesting microbes that are usually associated with obesity and diabetes in non-pregnant state, that is, increase in genera/species of Enterobacterioceae, Propionibacterium, Tenericutes, and an increased Firmicutes:Bacteroidetes ratio; but low in butyrogenic bacteria $-F$. praustnitzii, A. muciniphila and Bifidobacterium (Collado et al. 2008, Santacruz et al. 2010, Koren et al. 2012, Liu et al. 2017). Proteobacteria (Enterobacterioceae) is even suggested to be a predictive biomarker of diabetes (Amar et al. 2011). The increase in Bacteroides spp. and accompanying high LPS levels are also important processes that contribute to the inflammation.

Generally, the non-butyrate producers, which are coincidentally high LPS bearers, appear to be the proponents of this benign dysbiosis-associated proinflammatory state. Perhaps high LPS and low butyrate

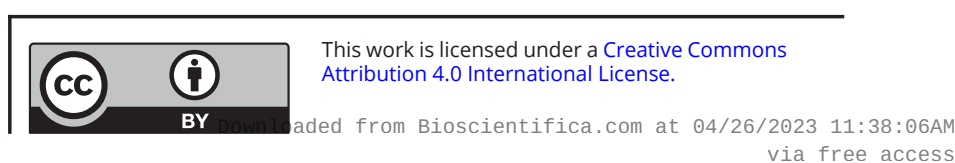


constitute the recipe that triggers this gestation-related benign diabetogenic state. Some authors suggest that maternal BMI prior to pregnancy is linked to the dysbiotic gut microbiota and inflammatory status at third trimester (Zacarías et al. 2018).

Of course, a dysbiotic gut microbiota deficient in butyrogenic bacteria and enriched with LPS bearers is associated with obesity and its comorbidities, which increase an individual's risk of pre-eclampsia and gestational diabetes. However, a similar microbialmetabolic profile commonly observed in the later stage of pregnancy does not produce the same harmful prognosis. The associated microbial composition and physiologic mechanistic pathways may be influenced by yet undetermined genetic and environmental factors. Additionally, genus and species level resolution of the hormone and inflammation-induced microbial changes could validate these observations and improve our understanding of the mechanisms involved. It may also elucidate the microbial, hormonal and/or immunologic 'threshold' for transition from the beneficial physiologic metabolic phenotype to pathologic states such as gestational diabetes, pre-eclampsia, etc.

In conclusion, though the gut microbiota influence host energy metabolism and homeostasis, and a dysbiosis is usually injurious to the host, the overall outcome of changes in gut microbiota structure and composition varies depending on the pregnancy status of the individual. The altered gut microbiota that connotes disease state in non-pregnant individual, promotes metabolic and immunological changes beneficial to the fetomaternal unit and success of pregnancy.

\section{Declaration of interest}

The authors declare that there is no conflict of interest that could be perceived as prejudicing the impartiality of this review.

\section{Funding}

Though this research did not receive any specific grant from any funding agency in the public, commercial or not-for-profit sector, E A and D A are funded by National Institute for Health Research (NIHR, 17/63/26).

\section{Author contribution statement}

Conceptualization and literature search - E A. Original draft preparation, review and editing - E A and D A. Both authors approved the final version of the manuscript for submission.

\section{Acknowledgements}

NIHR Global Health Research Group on PReterm birth prevention and manageMEnt (PRIME) at The University of Sheffield, UK; University of Cape Town, South Africa; University of Pretoria, South Africa; and The International Centre for Diarrhoeal Disease Research (ICDDR, B), Bangladesh (https://www. primeglobalhealth.co.uk).

\section{References}

Abee T, Klaenhammer TR \& Letellier L 1994 Kinetic studies of the action of lactacin F, a bacteriocin produced by Lactobacillus johnsonii that forms poration complexes in the cytoplasmic membrane. Applied and Environmental Microbiology 60 1006-1013. (https://doi.org/10.1128/AEM.60.3.1006-1013.1994)

Amabebe E \& Anumba DOC 2018 The vaginal microenvironment: the physiologic role of lactobacilli. Frontiers in Medicine 5 181. (https:// doi.org/10.3389/fmed.2018.00181)

Amabebe E \& Anumba DOC 2020 Female gut and genital tract microbiota-induced crosstalk and differential effects of short-chain fatty acids on immune sequelae. Frontiers in Immunology 112184. (https://doi.org/10.3389/fimmu.2020.02184)

Amabebe E, Robert FO, Agbalalah T \& Orubu ESF 2020 Microbial dysbiosis-induced obesity: role of gut microbiota in homoeostasis of energy metabolism. British Journal of Nutrition 123 1127-1137. (https://doi.org/10.1017/S0007114520000380)

Amar J, Serino M, Lange C, Chabo C, Iacovoni J, Mondot S, Lepage P, Klopp C, Mariette J, Bouchez O et al. 2011 Involvement of tissue bacteria in the onset of diabetes in humans: evidence for a concept. Diabetologia 54 3055-3061. (https://doi. org/10.1007/s00125-011-2329-8)

Bäckhed F, Manchester JK, Semenkovich CF \& Gordon JI 2007 Mechanisms underlying the resistance to diet-induced obesity in germ-free mice. PNAS 104 979-984. (https://doi.org/10.1073/ pnas.0605374104)

Barbour LA, McCurdy CE, Hernandez TL, Kirwan JP, Catalano PM \& Friedman JE 2007 Cellular mechanisms for insulin resistance in normal pregnancy and gestational diabetes. Diabetes Care 30 (Supplement 2) S112-S119. (https://doi.org/10.2337/dc07-s202)

Broskey NT, Wang P, Li N, Leng J, Li W, Wang L, Gilmore LA, Hu G \& Redman LM 2017 Early pregnancy weight gain exerts the strongest effect on birth weight, posing a critical time to prevent childhood obesity. Obesity 25 1569-1576. (https://doi.org/10.1002/ oby.21878)

Bruns CM \& Kemnitz JW 2004 Sex hormones, insulin sensitivity, and diabetes mellitus. ILAR Journal 45 160-169. (https://doi.org/10.1093/ ilar.45.2.160)

Buchanan TA 1991 Glucose metabolism during pregnancy: normal physiology and implications for diabetes mellitus. Israel Journal of Medical Sciences 27 432-441.

Caesar R, Reigstad CS, Bäckhed HK, Reinhardt C, Ketonen M, Östergren Lundén GÖ, Cani PD \& Bäckhed F 2012 Gutderived lipopolysaccharide augments adipose macrophage accumulation but is not essential for impaired glucose or insulin tolerance in mice. Gut 61 1701-1707. (https://doi.org/10.1136/ gutjnl-2011-301689)

Canfora EE, Jocken JW \& Blaak EE 2015 Short-chain fatty acids in control of body weight and insulin sensitivity. Nature Reviews: Endocrinology 11 577-591. (https://doi.org/10.1038/nrendo.2015.128)

Cani PD, Osto M, Geurts L \& Everard A 2012 Involvement of gut microbiota in the development of low-grade inflammation and type 2 diabetes associated with obesity. Gut Microbes 3 279-288. (https:// doi.org/10.4161/gmic.19625) https://raf.bioscientifica.com

https://doi.org/10.1530/RAF-20-0034 (c) 2021 The authors Published by Bioscientifica Ltd

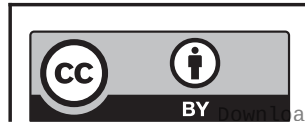

This work is licensed under a Creative Commons Attribution 4.0 International License. 
Chakraborti CK 2015 New-found link between microbiota and obesity World Journal of Gastrointestinal Pathophysiology 6 110-119. (https:// doi.org/10.4291/wjgp.v6.i4.110)

Chambers ES, Preston T, Frost G \& Morrison DJ 2018 Role of gut microbiota-generated short-chain fatty acids in metabolic and cardiovascular health. Current Nutrition Reports 7 198-206. (https:// doi.org/10.1007/s13668-018-0248-8)

Chen J \& Vitetta L 2020 Butyrate in inflammatory bowel disease therapy. Gastroenterology 158 1511. (https://doi.org/10.1053/j. gastro.2019.08.064)

Clarke SF, Murphy EF, Nilaweera K, Ross PR, Shanahan F, O'Toole PW \& Cotter PD 2012 The gut microbiota and its relationship to diet and obesity: new insights. Gut Microbes $\mathbf{3}$ 186-202. (https://doi.org/10.4161/gmic.20168)

Clavel T, Desmarchelier C, Haller D, Gérard P, Rohn S, Lepage P \& Daniel H 2014 Intestinal microbiota in metabolic diseases: from bacterial community structure and functions to species of pathophysiological relevance. Gut Microbes 5 544-551. (https://doi. org/10.4161/gmic.29331)

Collado MC, Isolauri E, Laitinen K \& Salminen S 2008 Distinct composition of gut microbiota during pregnancy in overweight and normal-weight women. American Journal of Clinical Nutrition $\mathbf{8 8}$ 894-899. (https://doi.org/10.1093/ajcn/88.4.894)

Cotillard A, Kennedy SP, Kong LC, Prifti E, Pons N, Le Chatelier E, Almeida M, Quinquis B, Levenez F, Galleron N et al. 2013 Dietary intervention impact on gut microbial gene richness. Nature 500 585-588. (https://doi.org/10.1038/nature12480)

Cox MA, Jackson J, Stanton M, Rojas-Triana A, Bober L, Laverty M, Yang X, Zhu F, Liu J, Wang S et al. 2009 Shortchain fatty acids act as antiinflammatory mediators by regulating prostaglandin E(2) and cytokines. World Journal of Gastroenterology 15 5549-5557. (https://doi.org/10.3748/wjg.15.5549)

Crusell MKW, Hansen TH, Nielsen T, Allin KH, Rühlemann MC, Damm P, Vestergaard H, Rørbye C, Jørgensen NR, Christiansen OB et al. 2018 Gestational diabetes is associated with change in the gut microbiota composition in third trimester of pregnancy and postpartum. Microbiome 6 89. (https://doi. org/10.1186/s40168-018-0472-x)

Di Cianni G, Miccoli R, Volpe L, Lencioni C \& Del Prato S 2003 Intermediate metabolism in normal pregnancy and in gestational diabetes. Diabetes/Metabolism Research and Reviews 19 259-270. (https://doi.org/10.1002/dmrr.390)

Edwards SM, Cunningham SA, Dunlop AL \& Corwin EJ 2017 The maternal gut microbiome During pregnancy. American Journal of Maternal Child Nursing 42 310-317. (https://doi.org/10.1097/ NMC.0000000000000372)

Ferrocino I, Ponzo V, Gambino R, Zarovska A, Leone F, Monzeglio C, Goitre I, Rosato R, Romano A, Grassi G et al. 2018 Changes in the gut microbiota composition during pregnancy in patients with gestational diabetes mellitus (GDM). Scientific Reports 8 12216-12216. (https://doi.org/10.1038/s41598-018-30735-9)

Fu J, Bonder MJ, Cenit MC, Tigchelaar EF, Maatman A, Dekens JA, Brandsma E, Marczynska J, Imhann F, Weersma RK et al. 2015 The gut microbiome contributes to a substantial proportion of the variation in blood lipids. Circulation Research 117 817-824. (https://doi.org/10.1161/ CIRCRESAHA.115.306807)

Garcia-Mantrana I \& Collado MC 2016 Obesity and overweight: impact on maternal and milk microbiome and their role for infant health and nutrition. Molecular Nutrition and Food Research 60 1865-1875. (https://doi.org/10.1002/mnfr.201501018)

Gohir W, Whelan FJ, Surette MG, Moore C, Schertzer JD \& Sloboda DM 2015 Pregnancy-related changes in the maternal gut microbiota are dependent upon the mother's periconceptional diet. Gut Microbes 6 310-320. (https://doi.org/10.1080/19490976.2015.10 86056)
Gregor MF \& Hotamisligil GS 2011 Inflammatory mechanisms in obesity. Annual Review of Immunology 29 415-445. (https://doi. org/10.1146/annurev-immunol-031210-101322)

Hod M, Kapur A, Sacks DA, Hadar E, Agarwal M, Di Renzo GC, Roura LC, McIntyre HD, Morris JL \& Divakar H 2015 (FIGO) Initiative on gestational diabetes mellitus: a pragmatic guide for diagnosis, management, and care. International Journal of Gynecology and Obstetrics 131 S173-S211.

Kaur H, Merchant M, Haque MM \& Mande SS 2020 Crosstalk between female gonadal hormones and vaginal microbiota across various phases of women's gynecological lifecycle. Frontiers in Microbiology 11 551. (https://doi.org/10.3389/fmicb.2020.00551)

Kirwan JP, Hauguel-De Mouzon S, Lepercq J, Challier JC, Huston-Presley L, Friedman JE, Kalhan SC \& Catalano PM 2002 TNF- $\alpha$ is a predictor of insulin resistance in human pregnancy. Diabetes 51 2207-2213. (https://doi.org/10.2337/diabetes.51.7.2207)

Kobyliak N, Virchenko o \& Falalyeyeva T 2016 Pathophysiological role of host microbiota in the development of obesity. Nutrition Journal 15 43. (https://doi.org/10.1186/s12937-016-0166-9)

Koh A, De Vadder F, Kovatcheva-Datchary P \& Bäckhed F 2016 From dietary fiber to host physiology: short-chain fatty acids as key bacterial metabolites. Cell 165 1332-1345. (https://doi.org/10.1016/j. cell.2016.05.041)

Koren O, Goodrich JK, Cullender TC, Spor A, Laitinen K, Bäckhed HK, Gonzalez A, Werner JJ, Angenent LT, Knight R et al. 2012 Host remodeling of the gut microbiome and metabolic changes during pregnancy. Cell 150 470-480.

Lain KY \& Catalano PM 2007 Metabolic changes in pregnancy. Clinical Obstetrics and Gynecology 50 938-948. (https://doi. org/10.1097/GRF.0b013e31815a5494)

Lazar V, Ditu LM, Pircalabioru GG, Picu A, Petcu L, Cucu N \& Chifiriuc MC 2019 Gut microbiota, host organism, and diet trialogue in diabetes and obesity. Frontiers in Nutrition 6 21. (https:// doi.org/10.3389/fnut.2019.00021)

Liu J, Yang H, Yin Z, Jiang X, Zhong H, Qiu D, Zhu F \& Li R 2017 Remodeling of the gut microbiota and structural shifts in preeclampsia patients in South China. European Journal of Clinical Microbiology and Infectious Diseases 36 713-719. (https://doi. org/10.1007/s10096-016-2853-z)

Lopetuso LR, Scaldaferri F, Petito V \& Gasbarrini A 2013 Commensal clostridia: leading players in the maintenance of gut homeostasis. Gut Pathogens 5 23. (https://doi.org/10.1186/17574749-5-23)

Mancuso G, Midiri A, Biondo C, Beninati C, Gambuzza M, Macrì D, Bellantoni A, Weintraub A, Espevik T \& Teti G 2005 Bacteroides fragilis-derived lipopolysaccharide produces cell activation and lethal toxicity via toll-like receptor 4. Infection and Immunity $\mathbf{7 3}$ 5620-5627. (https://doi.org/10.1128/IAI.73.9.5620-5627.2005)

Mesa MD, Loureiro B, Iglesia I, Gonzalez SF, Llurba Olive E, Garcia Algar O, Solana MJ, Cabero Perez MJ, Sainz T, Martinez L et al. 2020 The evolving microbiome from pregnancy to early infancy: a comprehensive review. Nutrients 12 133. (https:// doi.org/10.3390/nu12010133)

Mirmonsef P, Gilbert D, Zariffard MR, Hamaker BR, Kaur A, Landay AL \& Spear GT 2011 The effects of commensal bacteria on innate immune responses in the female genital tract. American Journal of Reproductive Immunology 65 190-195. (https://doi. org/10.1111/j.1600-0897.2010.00943.x)

Mirmonsef P, Zariffard MR, Gilbert D, Makinde $H$, Landay AL \& Spear GT 2012 Short-chain fatty acids induce pro-inflammatory cytokine production alone and in combination with toll-like receptor ligands. American Journal of Reproductive Immunology 67 391-400. (https://doi.org/10.1111/j.1600-0897.2011.01089.x)

Mor G \& Cardenas I 2010 The immune system in pregnancy: a unique complexity. American Journal of Reproductive Immunology 63 425-433. (https://doi.org/10.1111/j.1600-0897.2010.00836.x)

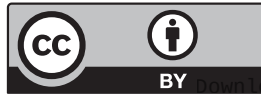

This work is licensed under a Creative Commons Attribution 4.0 International License. 
Mukhopadhya I, Hansen R, El-Omar EM \& Hold GL 2012 IBD what role do Proteobacteria play? Nature Reviews: Gastroenterology and Hepatology 9 219-230. (https://doi.org/10.1038/nrgastro.2012.14)

Mulak A, Taché Y \& Larauche M 2014 Sex hormones in the modulation of irritable bowel syndrome. World Journal of Gastroenterology 20 2433-2448. (https://doi.org/10.3748/wjg.v20. i10.2433)

Nelson SM, Matthews P \& Poston L 2010 Maternal metabolism and obesity: modifiable determinants of pregnancy outcome. Human Reproduction Update 16 255-275. (https://doi.org/10.1093/humupd/ dmp050)

Niu Q, Li P, Hao S, Zhang Y, Kim SW, Li H, Ma X, Gao S, He L, Wu W et al. 2015 Dynamic distribution of the gut microbiota and the relationship with apparent crude fiber digestibility and growth stages in pigs. Scientific Reports 5 9938. (https://doi.org/10.1038/ srep09938)

Nuriel-Ohayon M, Neuman H \& Koren O 2016 Microbial changes during pregnancy, birth, and infancy. Frontiers in Microbiology $\mathbf{7}$ 1031-1031. (https://doi.org/10.3389/fmicb.2016.01031)

Parada Venegas D, De la Fuente MK, Landskron G, González MJ, Quera R, Dijkstra G, Harmsen HJM, Faber KN \& Hermoso MA 2019 Short chain fatty acids (SCFAs)-mediated gut epithelial and immune regulation and its relevance for inflammatory bowel diseases. Frontiers in Immunology 10 277. (https://doi. org/10.3389/fimmu.2019.00277)

Ponzo V, Fedele D, Goitre I, Leone F, Lezo A, Monzeglio C, Finocchiaro C, Ghigo E \& Bo S 2019 Diet-gut microbiota interactions and gestational diabetes mellitus (GDM). Nutrients $\mathbf{1 1}$ 330. (https://doi.org/10.3390/nu11020330)

Preidis GA, Ajami NJ, Wong MC, Bessard BC, Conner ME \& Petrosino JF 2015 Composition and function of the undernourished neonatal mouse intestinal microbiome. Journal of Nutritional Biochemistry 26 1050-1057. (https://doi.org/10.1016/j. jnutbio.2015.04.010)

Pridmore RD, Berger B, Desiere F, Vilanova D, Barretto C, Pittet AC, Zwahlen MC, Rouvet M, Altermann E, Barrangou R et al. 2004 The genome sequence of the probiotic intestinal bacterium Lactobacillus johnsonii NCC 533. PNAS 101 2512-2517. (https://doi.org/10.1073/pnas.0307327101)

Prince AL, Antony KM, Ma J \& Aagaard KM 2014 The microbiome and development: a mother's perspective. Seminars in Reproductive Medicine 32 14-22. (https://doi.org/10.1055/s-0033-1361818)

Qin Y, Roberts JD, Grimm SA, Lih FB, Deterding LJ, Li R, Chrysovergis K \& Wade PA 2018 An obesity-associated gut microbiome reprograms the intestinal epigenome and leads to altered colonic gene expression. Genome Biology 19 7-7. (https://doi. org/10.1186/s13059-018-1389-1)

Ridaura VK, Faith JJ, Rey FE, Cheng J, Duncan AE, Kau AL, Griffin NW, Lombard V, Henrissat B, Bain JR et al. 2013 Gut microbiota from twins discordant for obesity modulate metabolism in mice. Science 341 1241214. (https://doi.org/10.1126/ science.1241214)

Rivière A, Selak M, Lantin D, Leroy F \& De Vuyst L 2016 Bifidobacteria and butyrate-producing colon bacteria: importance and strategies for their stimulation in the human gut. Frontiers in Microbiology 7 979. (https://doi.org/10.3389/fmicb.2016.00979)
Saad MJ, Santos A \& Prada PO 2016 Linking gut microbiota and inflammation to obesity and insulin resistance. Physiology $\mathbf{3 1}$ 283-293. (https://doi.org/10.1152/physiol.00041.2015)

Samson SL \& Garber AJ 2014 Metabolic syndrome. Endocrinology and Metabolism Clinics of North America 43 1-23. (https://doi. org/10.1016/j.ecl.2013.09.009)

Santacruz A, Collado MC, García-Valdés L, Segura MT, MartínLagos JA, Anjos T, Martí-Romero M, Lopez RM, Florido J, Campoy C et al. 2010 Gut microbiota composition is associated with body weight, weight gain and biochemical parameters in pregnant women. British Journal of Nutrition 104 83-92. (https://doi. org/10.1017/S0007114510000176)

Silva JPB, Navegantes-Lima KC, Oliveira ALB, Rodrigues DVS, Gaspar SLF, Monteiro VVS, Moura DP \& Monteiro MC 2018 Protective mechanisms of butyrate on inflammatory bowel disease. Current Pharmaceutical Design 24 4154-4166. (https://doi.org/10.2174 /1381612824666181001153605)

Stanislawski MA, Dabelea D, Wagner BD, Sontag MK, Lozupone CA \& Eggesbo M 2017 Pre-pregnancy weight, gestational weight gain, and the gut microbiota of mothers and their infants. Microbiome 5 113-113. (https://doi.org/10.1186/s40168-0170332-0)

Tester R \& Al-Ghazzewi FH 2018 Intrinsic and extrinsic carbohydrates in the vagina: a short review on vaginal glycogen. International Journal of Biological Macromolecules 112 203-206. (https://doi. org/10.1016/j.ijbiomac.2018.01.166)

Tungland B 2018 Chapter 2: Short-chain fatty acid production and functional aspects on host metabolism. In Human Microbiota in Health and Disease, pp. 37-106. Ed B Tungland. Academic Press.

Turnbaugh PJ, Ridaura VK, Faith JJ, Rey FE, Knight R \& Gordon JI 2009 The effect of diet on the human gut microbiome: a metagenomic analysis in humanized gnotobiotic mice. Science Translational Medicine 1 6ra14. (https://doi.org/10.1126/ scitranslmed.3000322)

Verdam FJ, Fuentes S, de Jonge C, Zoetendal EG, Erbil R, Greve JW, Buurman WA, de Vos WM \& Rensen SS 2013 Human intestinal microbiota composition is associated with local and systemic inflammation in obesity. Obesity 21 E607-E615. (https://doi.org/10.1002/oby.20466)

Vyas N, Nair S, Rao M \& Miraj SS 2019 Chapter 29: Childhood obesity and diabetes: role of probiotics and prebiotics. In Global Perspectives on Childhood Obesity, 2nd ed., pp. 363-376. Ed D Bagchi. Academic Press.

Walters WA, Xu Z \& Knight R 2014 Meta-analyses of human gut microbes associated with obesity and IBD. FEBS Letters $\mathbf{5 8 8}$ 4223-4233. (https://doi.org/10.1016/j.febslet.2014.09.039)

Xu Y, Wang N, Tan HY, Li S, Zhang C \& Feng Y 2020 Function of Akkermansia muciniphila in obesity: interactions with lipid metabolism, immune response and gut systems. Frontiers in Microbiology 11 219. (https://doi.org/10.3389/fmicb.2020.00219)

Zacarías MF, Collado MC, Gómez-Gallego C, Flinck H, Aittoniemi J, Isolauri E \& Salminen S 2018 Pregestational overweight and obesity are associated with differences in gut microbiota composition and systemic inflammation in the third trimester. PLOS ONE 13 e0200305. (https://doi.org/10.1371/journal. pone.0200305)

Received in final form 7 November 2020

Accepted 8 December 2020

Accepted Manuscript published online 14 December 2020 https://raf.bioscientifica.com

https://doi.org/10.1530/RAF-20-0034 (c) 2021 The authors Published by Bioscientifica Ltd

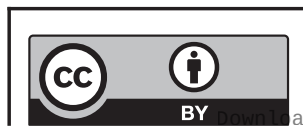

This work is licensed under a Creative Commons Attribution 4.0 International License. 